\title{
Short $\mathbb{C}$ ommunication
}

\section{Synovial Sarcoma of the Head and Neck - A Report of 3 Cases}

\author{
Produl Hazarika, Professor and Head, Parul Shah. Post doctoral Fellow. \\ Kailesh Pujary, Post doctoral Fellow, Balakrishnan R, Assistant Professor. \\ Department of Ent-head and neck surgery, Kasturba Medical College, \\ Manipal 576119,Karnataka, India.
}

\begin{abstract}
Synovial sarcoma is very rare and highly malignant tumour of the head and neck. Only 3 cases of synovial sarcoma have been treated 1980-1997 in Kasturba Medical College, Manipal. Treatment involved extensive surgical resection of the tumour (with neck dissection in two cases); and post-operative radiotherapy and chemotherapy in one case. Behaviour of the tumour was followed up till August 1997. It remains in our experience that synovial sarcoma, though a tumour or poor prognosis if treated by adequate tumour resection and adjunctive radiotherapy and chemotherapy, has good patient survival.
\end{abstract}

Key words

synovial sarcoma: laryngopharynx; parapharyngeal space

\section{INTRODUCTION}

$S_{b y}^{\text {in }}$

ince the first description of synovial sarcoma Sby Pack and Ariel in 1950, many cases have been reported. Jernstorm, in 1954, was the first to report this malignancy in the head and neck region. Synovial sarcoma accounts for 3 to $10 \%$ of all sarcomas and occurs predominantly in the extremities (Carrillo et al. 1992). Rare cases have been reported in the abdominal and chest wall (Roth et al, 1975).

Though synovial sarcoma arise by synovioblastic differentiation of connective tissue, it has also been found to arise in areas antomically devoid of synovioblastic tissue. In the head and neck, the tumour may be located high in the superior aspect of the neck, just beneath the mandible, in the prevertebral area from the base of the skull to the hypopharynx, in the retropharyngeal and parapharyngeal ar- eas and in the anterior neck along the border of sternocleidomastoid muscle as "orofacial" and "larngeal" sites (Roth et al, 1975).

\section{Case report 1}

In 1980, a 24 year old male presented with stridor of 10 days and history of dysphagia and change in voice of 4 months duration. Examination of the oropharynx revealed a round mass covered by white slough. The origin of the mass could not be appreciated even under general anaesthesia. It was firm in consistency occupying the whole of the hypopharynx and the oropharynx, with diffuse attachment to the medial wall of the right pyriform fossa.

A lateral view X-ray of the soft tissue neck showed a diffuse homogeneous soft tissue mass in the region or oropharnx and hypopharynx, obliterating the normal translucency. There was 
no evidence of calcification. Computerised tomography and magnetic resonance imaging facilities were not available for assessing the site and extent of the lesion. A punch biopsy taken from the growth was inconclusive.

Following an emergency tracheostomy, total excision of the mass was done through a translingual pharyngotomy (Trotter's) approach, after considering the position and extent of the growth. Incision extended from the midpoint of the lower lip to the upper border of the thyroid cartilage. The mandible, tongue and hyoid bone were divided in the midline. The exposed mass was found to be arising from the medial wall of the right pyriform fossa near the cricoarytenoid joint and was attached to the posterior wall of the hypopharynx. The larynx was pushed to the opposite side and compressed by the growth without being involved. The mass was excised completely. The postoperative period was uneventful. There had been no evidence of distant metastasis or recurrence for 10 years after which the patient was lost for follow up.

The gross specimen of the tumour mass measured $10 \times 10 \times 7.5 \mathrm{cms}$. and was covered with slough. Cut section was greyish in color and revealed areas of necrosis. Histopathological examination showed sheets of spindle cells with hyperchromatic nuclei and mitotic figures suggestive of fibrosarcoma. Due to the rarity of the tumour, we sought another opinion from Batsakis M.A. from Maine Medical Centre Portland and he diagnosed it as monophasic synovial sarcoma (Figure 1) (Hazarika et al, 1983).

There was no evidence of recurrence when seen ten years after treatment. The patient was lost to follow up thereafter.

\section{Case report 2}

In November 1987, a 30 year old male came with history of right side neck swelling since 8 months. It was insidious in onset and gradually progressive. On examination of the throat, a

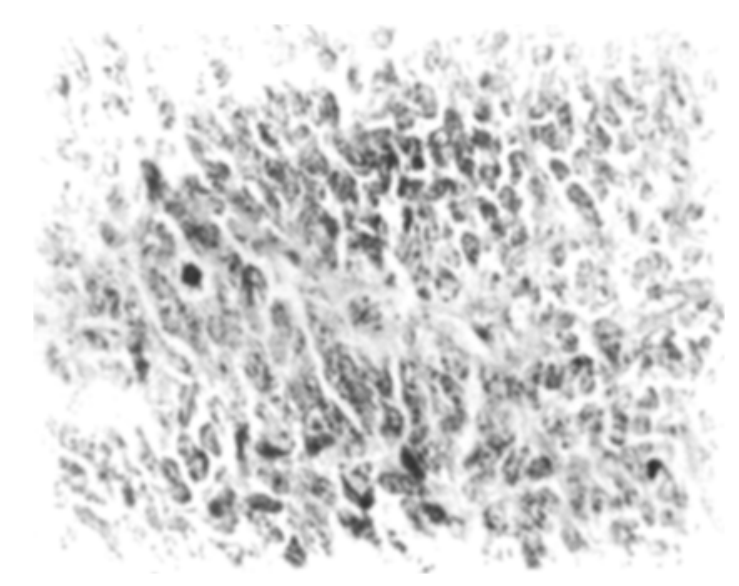

Fig 1. Monophasic variety of synovial sarcoma, showing nests of polygonal cells with large nuclei and occasional $\mathrm{mi-}$ totic figures $(H \& E X 400)$.

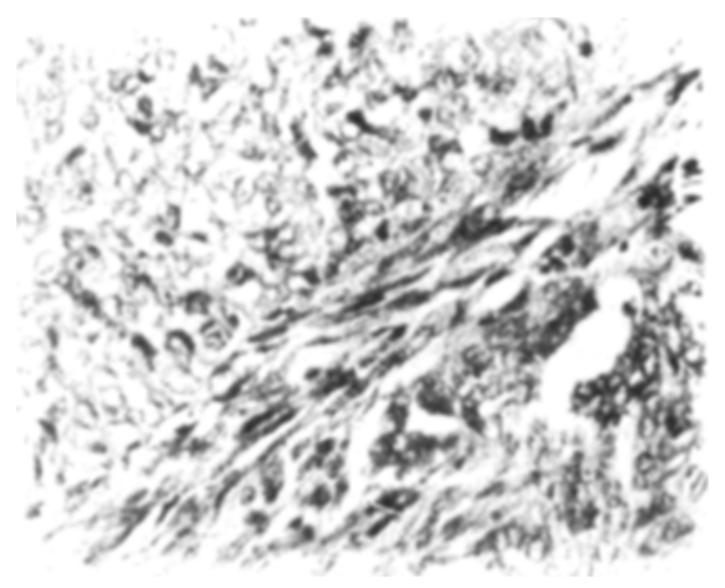

Fig 2. Biphasic variety of synovial sarcoma, showing plump spindle cells arranged in fascicles along with nests of polygonal cells with large round nuclei $(H \&$ EX 400).

swelling was seen behind the right posterior pillar extending to the right vallecula. The right tonsil was pushed medially and there was wasting of the right side of the tongue. A spherical swelling was noted on the right side of the neck extending from the angle of the mandible to almost the midline. It was firm in consistency, non-tender with restricted mobility, and ballotable. 
Routine blood and urine investigations were within normal limits. The patient was on antidiabetic treatment. A.C.T. scan of the neck confirmed the diagnosis of right parapharyngeal mass displacing the carotid sheath posteriorly without infiltrating it.

In March 1988, the parpharyngeal tumour was excised by a transcervical transmandibular approach along with a right modified neck dissection.

The tumour mass was $6 \times 5 \times 4 \mathrm{cms}$., dark brown in color, well encapsulated with circumscribed lobulated areas. Cut section showed areas of haemorrhage and necrosis. A biphasic variety of synovial sarcoma was diagnosed on histopathology (fugure 2). The neck dissection specimen showed no significant pathology.

Post-operative radiotherapy was planned for MTD of 5000 cGys in 25 fractions over 35 days with 2 courses of chemotherapy of vincristine $2 \mathrm{mg}$, actinomycin D $1 \mathrm{mg}$ and cyclophosphamide $500 \mathrm{mg}$.

However, the patient tolerated 3800 cGys in 19 fractions with complete course of chemotherapy. The patient has maintained a regular yearly follow up for 9 years with no evidence of locoregional recurrence or distant metastasis.

\section{Case report 3}

A 20 year old female presented with history of dysphagia, odynophagia and change in voice of one and a half years duration when she reported in August 1993. The complaints were insidious in onset, more to solids than liquids and gradually increasing. She gave history of vomiting out few bluish grey bits and pieces following which her symptoms improved. Prior management at another center was as follows: a tracheostomy was done for stridor $11 / 2$ years back. A course of radiotherapy of 300 cGys over 15 days was given and was stopped as there was no improvement. Two subsequent biopsies at 6 and 10 months were reported as fibroma and hemangiopericytoma, respectively. The patient was referred to us for further management.
On examination, a $5 \times 3 \mathrm{~cm}$. bluish grey mass was seen protruding into oropharyn $x$ from below reaching upto the base tongue. It was covered with slough, firm, non-tender and did not bleed on touch. Rigid telescopy revealed, a smooth growth arising from right aryepiglottic fold and right arytenoid. Right ventricular band and aryepiglottic fold were odematous and right hemilarynx fixed.

A "Widefield" laryngectomy (Figure 3) with right hemithyroidectomy and right radical neck dissection was done following a complete preoperative work up. The post-operative period was uneventful.

Histopathological section of the tumour showed a highly vascular tumour; spindle shaped cells arranged in whorls and fascicles showing high mitotic activity. These features were suggestive of a monophasic fibroblastic variety of synovial sarcoma. The resected margins were free and the lymph nodes and soft tissue showed no evidence of metastasis.

In August 1996, she underwent surgical voice restoration by tracheo-oesophageal puncture and at present is using the Blom Singers voice prosthesis. On five years of follow up there was no evidence of recurrence.

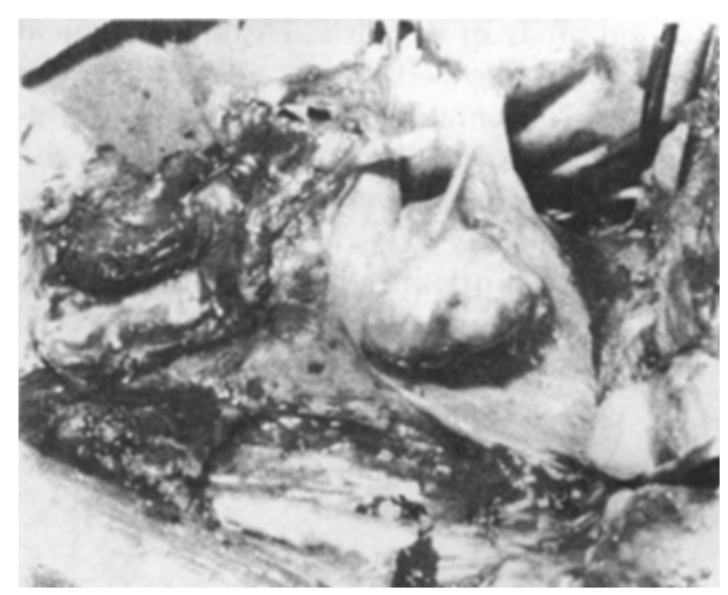

Fig 3. Intraoperative picture of Widefield laryngectomy (case 3), showing a smooth mass arising from the aryepiglottic fold and arytenoid. 


\section{Discussion}

On review of approximately 15,500 cases of head and neck malignancies in our department, from 1980 to 1997, a total of 3 cases were reported as synovial sarcoma. A more recent report by Enzinger and Weiss from the Armed Force Institute of Pathology [AFIP] reviewed 345 cases of synovial sarcoma including 31 originating in the head and neck 19\%] (Enzinger and Weiss, 1988). The AFIP reported that most of their synovial sarcomas were located in the cervical and hypopheryngeal regions.

In 1982. Applebaum reviewed the English and European literature and fewer than 50 cases of synovial sarcoma in the head and neck were noted, 21 of these tumours were in the hypopharynx (Quinn, 1984). Our study had 2 cases of synovial sarcoma in the laryngopharynx arising from the pyriform fossa (Case 1) and the arytenoid and aryepiglottic fold (Case 2).

Roth et al observed 24 cases of synovial sarcoma encountered in the parapharyngeal region and the region of anterior neck from 1948-1969 (Roth et al, 1975). Unlike patients with synovial sarcoma elsewhere who present with symptoms after several years, most patients with head and neck lesions seek medical attention within a year of onset of their symptoms.

The classical synovial sarcoma is unique tumour which in reality is a true carcinosarcoma. Characteristic macroscopic appearance is of pseudocapsulated, greyish or fleshy coloured mass with solid, cystic or mixed pattern and areas of haemorrhage and necrosis. A variable degree of vascularity is present, ranging from numerous dilated vascular spaces resembling hemangiopricytoma to a few scattered vascular spaces. Enzinger and Weiss classified synovial sarcoma into 4 groups based on morphological characteristics: the biphasic pattern containing recognizable epithelial and arcomatous component constituting $55 \%$, the monophasic form which manifests the sarcoma in $39 \%$ and monophasic epi- thelial in $6 \%$. The poorly differentiated synovial sarcoma are rare (Enzinger and Weiss, 1988).

C.T. scan should be done to assess erosive or destructive bone changes particularly in the orofacial region; a heterogeneous pattern with significant contrast enhancement is seen. Calcification is another characteristic feature that is present in approximately $30 \%$ of synovial sarcoma and is a good prognostic sign (Sigal et al, 1992).

The clinical differential diagnosis at presentation include meningioma, neurilemmoma, lymphoma, cartilaginous tumour, chondrosarcoma, nasopharyngeal carcinoma and sarcoidosis, rhabdomyosarcoma in children. mucormycosis in diabetics, aspergillosis in immunocompromised patients (Miloro et al, 1994). Immunocytochemistry and electron microscopy are helpful in distinguishing from salivary gland tumours, carcinomas, neurogenic sarcomas, hemangiopericytoma and fibrosarcoma. In synovial sarcoma, cytokeratin stains positive for epithelial cells and vimentin stains positive in spindle cells ( $\mathrm{Pal}$ et al, 1993).

Cases with lung metastasis may reappear 35 years after initial therapy and rarely pulmonary metastasis may present before the primary tumour is detected. The incidence of metastasis in the series of Enzinger is $94 \%$ in the lungs, followed by $21 \%$ in lymph nodes and $9 \%$ in bone marrow (Enzinger and Weiss, 1988). In the opinion of Batsakis, head and neck synovial sarcomas do not appear to share this tendency for regional lymph node and osseous metastasis (Batsakis, 1979). Robinson, Destian and Hinton were the first to report invasion of the brain Robinson et al, 1994).

Currently, the treatment of choice appears to be a radical surgical resection and post operative radiotherapy (Gapanvicius et al, 1978). We treated two patients with modified neck dissection with NO neck. -rom histopatholo- gical evidence of the neck dissection specimens, it 
has been noted that regional metastasis is not important, but the prevention of distant metastasis should be attempted with chemotherapy. Our patient in whom a neck dissection was not done, there was no evidence of recurrence over 10 years of follow up.

A trial of adjuvant chemotherapy with vincristine, actinomycin-D and cyclophosphamide was given to one patient in our series. Lately, it has been suggested that doxorubicin may increase the survival rate of synovial sarcoma (Gatti et al, 1975). The exact role of chemotherapy remains underfined. A long term follow up is necessary because patient may have recurrence even after 5 years. Smaller tumours of less than $5 \mathrm{cms}$. and those with a clear biphasic pattern seem to have better prognosis (Hordgik, 1984).

In our experience, from the year 1980 to 1997, synovial sarcoma constitutes only a small percentage of head and neck malignancies. An aggressive local surgical excision is mandatory, following which radiotherapy and chemotherapy should be considered in view of the nature of the tumour.

\section{References}

1. Batsakis, J.G. (1979) Tumours of The Head and Neck clinical and pathological considerations, 2nd Edition, The Williams \& Wilkins Company, Baltimore, pp 350-368.

2. Carrillo, R., Peralto, J.L.R., Batsakis, J.C. (1992) Synovial sarcoma of the head and neck. Annals of Otology Rhinology and Laryngology 101(4): 367-370.

3. Enzinger, F.M., Weiss, S.W. (1988) Synovial Sarcoma. In Soft Tissue Tumours, 2nd edition The C.V. Mosby Company, St. Louis pp 659-688.

4. Gapanvicius, B.G., Behar, A.J., Chisin, R. (1978) Synovial Sarco ma of the hypopharynx. Annals of Otology Rhinology and Laryngology 87: 356-359.

5. Gatti, W.M., Strom, C.G., Orfei, E. (1975) Synovial sarcoma of the laryngopharynx. Archives Otolaryngology 101: 633-636.

6. Hazarika, P., Revikumar, Nayak, RG. (1983) Synovial Sarcoma of Hypoharynx. Indian Journal of Otolaryngology 35(4):97-98.

7. Hordgik, G.J. (1984) Synovial sarcoma of the pharynx. Ear Nose, and Throat Journal 63: 597598.

8. Jernstrom, P. (1954) Synovial sarcoma of the pharynx. American Journal of Clinical Pathology 24: 957.

9. Miloro, M., Quinn, P.D., Jeffrey, C.B., Stewart (1994) Monophasic spindle cell sarcoma of the head and neck. Report of two cases and review of the literature. Journal Oral and Maxillofacial Surgery 52: 309-313.

10. Pack, G.T., Ariel, I.M. (1950) Synovial sarcoma (malignant synovioma): A report of 60 cases. Surgery 28: 1047.

11. Pal, S., Chinoy, R., Pradhan, S.A., D'Cruz, A.K., Kane, S.V., Yadav, J.N. (1993) Head And Neck Synovial Sarcomas. Journal of Surgical Oncology 54: 82-86. 
12. Palmer, B.V., Levene, A., Shaw, H.J. (1983) Synovial sarcoma of the pharynx and oesophagus. The Journal of Laryngology and Otology 97: 1173-1176.

13. Quinn, H.J. (1984) Synovial Sarcoma of the larynx treated by Partial Laryngectomy. Laryngoscope 94: 1158-1161.

14. Robinson, D.L., Destian, S., Hinton, D.R. (1994) Synovial sarcoma of the neck: Radiographaic findings with a review of literature. American Journal of Otolaryngology 15(1): 46-53.

15. Roth, J.A., Enzinger, F.M., Tannenbaum, M. (1975) Synovial sarcoma of the neck: A follow up study of 24 cases. Cancer 35: 1243-1253.

16. Sigal, R., Dominique, M. Luloinski, B., Shapero, L.G., Bosq, J., Vanel, D. (1992) Pathology consultation synovial sarcoma of the head and neck. Annals of Otology, Rhinology and Laryngology 101: 367-370. 